\title{
Sums of Powers of Integers and Bernoulli Numbers Clarified
}

\author{
Do Tan $\mathrm{Si}^{1}$ \\ ${ }^{1}$ HoChiMinhcity Physical Association, Vietnam, 40 Dong Khoi, Q1, HochiMinhcity, Vietnam \\ Correspondence: Do Tan Si, HoChiMinhcity Physical Association, Vietnam, 40 Dong Khoi, Q1, HochiMinhcity, \\ Vietnam. E-mail: tansi_do@yahoo.com \\ Received: December 11, 2016 \\ doi:10.5539/apr.v9n2p12 \\ Accepted: January 21, 2017 \\ Online Published: February 16, 2017 \\ URL: https://doi.org/10.5539/apr.v9n2p12
}

\begin{abstract}
This work exposes a very simple method for calculating at the same time the sums of powers of the first integers $S_{m}(n)$ and the Bernoulli numbers $B_{m}$. This is possible thank only to the relation $S_{m}(x+1)-S_{m}(x)=x^{m}$ and the Pascal formula concerning $S_{m}(n)$ which may be explained as if the vector $\left|n^{2}-n, n^{3}-n, \ldots, n^{m+1}-n\right|$ is the transform of the vector $\left|S_{1}(n), S_{2}(n), \ldots, S_{m}(n)\right|$ by a matrix $P$ built from the Pascal triangle. Very useful relations between the sums $S_{m}(n)$, the Bernoulli numbers $B_{m}$ and elements of the inverse matrix of $P$ are deduced, leading straightforwardly to known and new properties of them.
\end{abstract}

\section{Introduction}

From the time Faulhaber (1631) published the formulae for calculating the first seventeen sums of powers of first integers and during nearly four centuries, many attempts for proving and extending them are performed, for example by Fermat, Pascal, Bernoulli, etc... as we can see in the works of Beery (2010), Coen (1996) and the references therein; then by many nowadays authors such as Edwards (1986), Knuth (1993), etc...

$$
S_{m}(n)=\sum_{k=1}^{n-1} k^{m}
$$

The results obtained are that $S_{m}(n)$ may be calculated from a $m$-terms recurrence relation or via a formula involving the Bernoulli numbers $B_{0}, B_{1}, \ldots, B_{m}$. The latters are calculated from its generating function or by a recurrence relation. We see that by these formulae the calculations of $S_{m}(n)$ and $B_{m}$ are not so easy for big $m$.

$$
\frac{t}{e^{t}-1}=\sum_{m=1}^{\infty} B_{m} \frac{t^{m}}{m !}
$$

In order to simplify the problem, we expose hereinafter an alternative method consisting of three steps.

Firstly we will utilize operator calculus to prove the matrix formula

$$
\left|\begin{array}{c}
S_{1}(n) \\
S_{2}(n) \\
S_{3}(n) \\
. . \\
S_{m}(n)
\end{array}\right|=P^{-1}\left|\begin{array}{c}
n^{2}-n \\
n^{3}-n \\
n^{4}-n \\
\\
n^{m+1}-n
\end{array}\right|
$$

where $P^{-1}$ is the inverse of the matrix $P_{i j}=\left(\begin{array}{c}i+1 \\ j\end{array}\right)$ formed from the Pascal triangle.

Secondly we utilize the well-known formula

$$
S_{m}(x+1)-S_{m}(x)=x^{m}
$$

in order to get the relations between the elements of $P^{-1}$ and a set of numbers $B_{m}$ defined as followed

$$
B_{m+1}=-(m+1) \int_{0}^{1} S_{m}(x) d x
$$

These relations permit the calculations of $S_{m}(n)$ and $B_{m}$ by a simple algorithm. 
Finally we prove that the so defined numbers $B_{m}$ verify the generating functions of the Bernoulli numbers and may conclude that this set of $B_{m}$ is exactly the set of Bernoulli numbers.

\section{Sums of Powers of Integers and the Pascal Matrix}

In order to calculate the sum

$$
S_{m}(n)=\sum_{k=1}^{n-1} k^{m}=1^{m}+2^{m}+\ldots+(n-1)^{m}
$$

let us consider the function

$$
S(x) \equiv e^{x}+e^{2 x}+\ldots+e^{(n-1) x}
$$

and the operator

$$
S\left(D_{x}\right) \equiv e^{D_{x}}+e^{2 D_{x}}+\ldots+e^{(n-1) D_{x}}
$$

constructed from the derivative operator $D_{x} \equiv \frac{d}{d x}$.

We have

$$
\begin{aligned}
& S^{\prime}\left(D_{x}\right) \equiv e^{D_{x}}+2 e^{2 D_{x}}+\ldots+(n-1) e^{(n-1) D_{x}} \\
& S^{\prime \prime}\left(D_{x}\right) \equiv e^{D_{x}}+2^{2} e^{2 D_{x}}+\ldots+(n-1)^{2} e^{(n-1) D_{x}} \\
& \ldots \\
& S^{(m)}\left(D_{x}\right) \equiv e^{D_{x}}+2^{m} e^{2 D_{x}}+\ldots+(n-1)^{m} e^{(n-1) D_{x}}
\end{aligned}
$$

From the fundamental identity in operator calculus (Do Tan Si 2016)

$$
f\left(D_{x}\right) g(\hat{X}) \equiv g(\hat{X}) f\left(D_{x}\right)+\ldots+\frac{1}{k !} g^{(k)}(\hat{X}) f^{(k)}\left(D_{x}\right)+\ldots
$$

where $\hat{X}$ is the Eckaert operator « multiply with the argument $x$ » we get

$$
S\left(D_{x}\right) \hat{X}^{m} \equiv \hat{X}^{m} S\left(D_{x}\right)+\left(\begin{array}{c}
m \\
1
\end{array}\right) \hat{X}^{m-1} S^{\prime}\left(D_{x}\right)+\ldots+\left(\begin{array}{c}
m \\
m
\end{array}\right) \hat{X}^{m-m} S^{(m)}\left(D_{x}\right)
$$

Applying this identity on a constant and utilizing the property of the translation operator

$$
e^{a D_{x}} f(x)=f(x+a)
$$

and the fact that

$$
S^{(k)}\left(D_{x}\right) 1=1+2^{k}+\ldots+(n-1)^{k}=S_{k}(n)
$$

we get the formula

$$
\begin{gathered}
S\left(D_{x}\right) x^{m}=x^{m} S\left(D_{x}\right) 1+\left(\begin{array}{c}
m \\
1
\end{array}\right) x^{m-1} S_{1}(n)+\ldots+\left(\begin{array}{c}
m \\
m
\end{array}\right) S_{m}(n) \\
(x+1)^{m}+\ldots+(x+n-1)^{m}=x^{m}(n-1)+\left(\begin{array}{c}
m \\
1
\end{array}\right) x^{m-1} S_{1}(n)+\ldots+\left(\begin{array}{c}
m \\
m
\end{array}\right) S_{m}(n)
\end{gathered}
$$

For $x=1$ it becomes

$$
\begin{gathered}
2^{m}+\ldots+n^{m}=(n-1)+\left(\begin{array}{c}
m \\
1
\end{array}\right) S_{1}(n)+\ldots+\left(\begin{array}{c}
m \\
m-1
\end{array}\right) S_{m-1}(n)+S_{m}(n) \\
n^{m}-n=\left(\begin{array}{c}
m \\
1
\end{array}\right) S_{1}(n)+\left(\begin{array}{c}
m \\
2
\end{array}\right) S_{2}(n)+\left(\begin{array}{c}
m \\
3
\end{array}\right) S_{3}(n)+\ldots+\left(\begin{array}{c}
m \\
m-1
\end{array}\right) S_{m-1}(n) \quad m>1
\end{gathered}
$$

i.e. for $m \geq 1$

$$
n^{m+1}-n=\left(\begin{array}{c}
m+1 \\
1
\end{array}\right) S_{1}(n)+\left(\begin{array}{c}
m+1 \\
2
\end{array}\right) S_{2}(n)+\ldots+\left(\begin{array}{c}
m+1 \\
m
\end{array}\right) S_{m}(n)
$$

We note that the above $m$-terms recurrence formula for calculating $S_{m}(n)$ had been proven by Pascal by an elaborated arithmetic one may find in (Beery, 2010).

It is not convenient to utilize for high values of $m$. 
In order to better exploit the formula (9), let us consider the matrix $P$ composed of elements of the Pascal triangle

$$
\begin{aligned}
& P_{\mathrm{ij}}=\left(\begin{array}{c}
i+1 \\
j
\end{array}\right) \text { for } i \geq j \\
& P_{\mathrm{ij}}=0 \text { for } i<j
\end{aligned}
$$

Hereinafter $P$ will be named Pascal matrix. With respect to $P$ we can write (9) under the matrix form

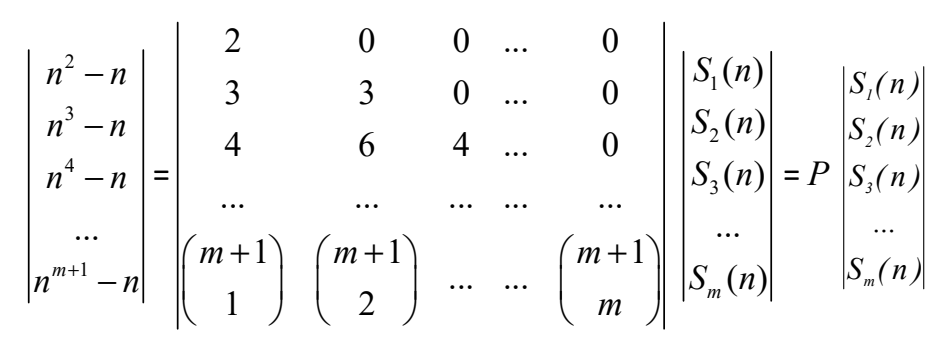

The inverse matrix $P^{-1}$ of $P$ has elements $P_{i j}^{-1}$ proportional to the determinant of the matrix obtained from $P$ by omitting the $j^{\text {th }}$ line and the $i^{\text {th }}$ column, i.e. the determinant of a matrix where all the elements situated at the right of the principal diagonal are equal to zero. Moreover if $i<j$ at least one element of the principal diagonal of $P^{-l}$ is equal to zero. It results that

$$
P^{-1}{ }_{i j}=0 \text { for } i<j
$$

Now because $P^{-1} P \equiv P P^{-1} \equiv \hat{I}$ we have the formula

$$
\left|\begin{array}{c}
S_{1}(n) \\
S_{2}(n) \\
S_{3}(n) \\
. . \\
S_{m}(n)
\end{array}\right|=P^{-1}\left|\begin{array}{c}
n^{2}-n \\
n^{3}-n \\
n^{4}-n \\
\ldots \\
n^{m+1}-n
\end{array}\right|=\left|\begin{array}{ccccc}
P^{-1}{ }_{11} & 0 & 0 & \ldots & 0 \\
P^{-1}{ }_{21} & P^{-1}{ }_{22} & 0 & \ldots & 0 \\
P^{-1}{ }_{31} & P^{-1}{ }_{32} & P^{-1} & \ldots & 0 \\
\ldots & \ldots & \ldots & \ldots & \ldots \\
P^{-1}{ }_{m 1} & P^{-1}{ }_{m 2} & & & P_{m m}^{-1}
\end{array}\right|\left|\begin{array}{c}
n^{2}-n \\
n^{3}-n \\
n^{4}-n \\
\ldots \\
n^{m+1}-n
\end{array}\right|
$$

On behalf of this formula we may state the important theorem linking $S_{m}(n)$ with $P^{-1}$ :

$$
\begin{aligned}
& \text { " } S_{m}(n) \text { is a polynomial of order } m+1 \text { in } n \text { having the form } \\
& S_{m}(n)=-\left(P_{m 1}^{-1}+P_{m 2}^{-1}+\ldots+P_{m m}^{-1}\right) n+P_{m 1}^{-1} n^{2}+\ldots+P_{m m}^{-1} n^{m+1} \text { » }
\end{aligned}
$$

This theorem gives the coefficients of $n, n^{2}, \ldots, n^{m+1}$ and justifies the fact that $S_{m}(1)=0$, i.e. the sum of all coefficients in $S_{m}(n)$ is equal to zero.

By extension we propose to take $0^{0}=1$ and define

$$
S_{0}(n)=0^{0}+1^{0}+. .+(n-1)^{0}=n
$$

so that coherently $S_{m}(0)=0$ for $m \geq 0$.

\section{Calculations of $P^{-1}$}

By (14) we see that for obtaining $S_{m}(n)$ one has to calculate the elements of $P^{-1}$.

(i) The matrix $P^{-1}$ may be obtained numerically by inversion of the Pauli matrix $P$ thank to a software.

(ii) It may also be obtained analytically by the following method leaned firstly on the fact that a polynomial of order $m$ which has more than $m$ roots is identically equal to zero.

In fact, let us firstly consider the polynomial

$$
Q_{m}(x)=S_{m}(x+1)-S_{m}(x)-x^{m}
$$

From (1) we have

$$
Q_{m}(n)=S_{m}(n+1)-S_{m}(n)-n^{m}=0 \quad \forall n=n_{1}, n_{2}, \ldots, n_{m}, n_{m+1}, \ldots . \text { integers }
$$

so that

$$
Q_{m}(x)=a_{m}\left(x-n_{1}\right)\left(x-n_{2}\right) \ldots\left(x-n_{m}\right)
$$




$$
Q_{m}\left(n_{m+1}\right)=0=a_{m}\left(n_{m+1}-n_{1}\right)\left(n_{m+1}-n_{2}\right) \ldots\left(n_{m+1}-n_{m}\right) \neq 0
$$

which is a paradox unless $Q_{m}(x)=0$.

From the above remark we have the equation

$$
S_{m}(x+1)-S_{m}(x)=x^{m}
$$

that Johnson (1986) have proven by another method.

Secondly, following the idea of Raugh (2014), we perform an integration over $x$ the above equation from 0 to $j$ and get

$$
\begin{gathered}
\int_{0}^{j} S_{m}(x+1)-\int_{0}^{j} S_{m}(x)=\frac{1}{m+1} j^{m+1} \\
\int_{1}^{j+1} S_{m}(x)-\int_{0}^{j} S_{m}(x)=\frac{1}{m+1} j^{m+1} \\
\int_{0}^{j+1} S_{m}(x)-\int_{0}^{j} S_{m}(x)-\int_{0}^{1} S_{m}(x)=\frac{1}{m+1} j^{m+1}
\end{gathered}
$$

Summation over $j$ from 1 to $(n-1)$ leads to the two-terms recurrence formula

$$
\int_{0}^{n} S_{m}(x)-n \int_{0}^{1} S_{m}(x)=\frac{1}{m+1} S_{m+1}(n)
$$

which allows us to calculate $S_{m+1}(x)$ once $S_{m}(x)$ known.

For examples

$$
\begin{aligned}
& S_{1}(n)=\int_{0}^{n} S_{0}(x)-n \int_{0}^{1} S_{0}(x)=\frac{n^{2}}{2}-\frac{n}{2} \\
& S_{2}(n)=2 \int_{0}^{n} S_{1}(x)-2 n \int_{0}^{1} S_{1}(x)=\frac{n^{3}}{3}-\frac{n^{2}}{2}-n\left(\frac{1}{3}-\frac{1}{2}\right)=\frac{n}{6}\left(2 n^{2}-3 n+1\right) \\
& S_{3}(n)=3 \int_{0}^{n} S_{2}(x)-3 n \int_{0}^{1} S_{2}(x)=\frac{n^{4}}{4}-\frac{n^{3}}{2}+\frac{n^{2}}{4}-n\left(\frac{1}{4}-\frac{1}{2}+\frac{1}{4}\right)=\frac{n^{2}}{4}(n-1)^{2}
\end{aligned}
$$

Thirdly, although (18) constitutes by itself a formula for obtaining sums of powers of integers established in 1631 by Faulhaber (Beery 2010) and beyond in a simple manner, we would like to utilize the theorem (14) to advance further in simplicity as explained hereafter.

(iii) Defining the set of numbers $B_{m}$ from the set of $S_{m}(x)$ as followed

$$
B_{m+1}=-(m+1) \int_{0}^{1} S_{m}(x)
$$

which gives for example

we get the very useful formula

$$
B_{1}=-\int_{0}^{1} S_{0}(n)=-\int_{0}^{1} n=-\frac{1}{2}
$$

$$
(m+1) \int_{0}^{n} S_{m}(x)+n B_{m+1}=S_{m+1}(n)
$$

From the theorem (14) we see that the polynomial $\int_{0}^{n} S_{m}(x)$ begins with a term in $n^{2}$ so that by equalizing the coefficients of $n$ in both members of (20) we get the first relation between $B_{m}$ and the $P^{-1}{ }_{m k}$

$$
B_{m+1}=-\sum_{j=1}^{m+1} P_{m+1 j}^{-1}
$$

Afterward, by equalizing the coefficients of $n^{2}$ and $n^{k+2}$ we get the interrelations between elements of $P^{-1}$ : 


$$
\begin{gathered}
\left(-\sum_{j=1}^{m} P_{m j}^{-1}\right) \int_{0}^{1} x=-\frac{1}{2} \sum_{j=1}^{m} P_{m j}^{-1}=\frac{1}{m+1} P_{m+11}^{-1} \\
P_{m k}^{-1} \int_{0}^{1} x^{k+1}=\frac{1}{k+2} P_{m k}^{-1}=\frac{1}{m+1} P_{m+1 k+1}^{-1}
\end{gathered}
$$

From (21) and (22) we get the relation linking $B_{m+1}$ with $P^{-1}{ }_{m+2} 1$

$$
B_{m+1}=-\sum_{i=1}^{m+1} P_{m+1 i}^{-1}=\frac{2}{m+2} P_{m+21}^{-1}
$$

or

$$
B_{m}=-\sum_{i=1}^{m} P_{m i}^{-1}=\frac{2}{m+1} P_{m+11}^{-1}
$$

An algorithm for calculating $P^{-1}$ is possible by observing that from (24), (23)

we get the formula

$$
B_{k}=\frac{2}{k+1} P_{k+11}^{-1}=\frac{2}{k+1} \frac{3}{k+2} P_{k+22}^{-1}=\ldots=\frac{(m+1) ! k !}{(k+m) !} P_{k+m m}^{-1}
$$

It may be described as followed

"Departing from

$$
P^{-1}{ }_{11}=\frac{1}{P_{11}}=\frac{1}{2}
$$

and utilizing (25) we get $B_{0}=2 P_{11}^{-1}=1$ then all the elements of the principal diagonal of $P^{-1}$ from $P_{11}^{-1}$ until $P_{m m}^{-1}$.

Afterward, by (24) we get $B_{1}=-P_{11}^{-1}=-\frac{1}{2}$ then by (25) all the elements of the first parallel with respect to the principal diagonal of $P^{-1}$ from $P_{21}^{-1}$ until $P_{m m-1}^{-1}$.

Once again, by (24) we get $B_{2}=-\left(P_{11}^{-1}+P_{21}^{-1}\right)$ then by (25) all the elements of the second parallel with respect to the principal diagonal of $P^{-1}$ from $P_{31}^{-1}$ until $P_{m m-2}^{-1}$.

Repeatedly, by (24) we get $B_{k}$ then by (25) all the elements of the $k^{\text {th }}$ parallel from $P_{k+11}^{-1}$ until $P_{m m-k}^{-1}$.

Finally, by (24) we get $B_{m-1}$ then by (25) the element

$$
P_{m 1}^{-1} . "
$$

This algorithm for calculating analytically all the $S_{m}(n)$ and $B_{m}$ via the matrix $P^{-1}$ needs few arithmetic operations comparing to the known methods.

By this reason it is exposed in this work. We note that by (25) we have in particular

$$
\begin{gathered}
(m+1) P_{m m}^{-1}=\ldots=2 P_{11}^{-1}=1 \\
P_{m m-1}^{-1}=\ldots=P_{21}^{-1}=B_{1}=-\frac{1}{2}
\end{gathered}
$$

We give in Appendix the first seventeen lines of $P^{-1}$. From this $17 \times 17$ submatrix one obtain the seventeen formulae deduced from the Faulhaber formulae (Beery 2010) for calculating $S_{1}(n), S_{2}(n), \ldots, S_{17}(n)$ and seventeen first values of $B_{m}$.

(iv) Another way to calculate the numbers $B_{m}$ once $P^{-1}$ known is possible from the relation (21) which leads to the matrix relation

where

$$
\vec{B}=-P^{-1} \overrightarrow{1}
$$




$$
\begin{aligned}
\vec{B} & \equiv\left|\begin{array}{llllll}
B_{1} & B_{2} & \ldots & \ldots & B_{m} & \ldots
\end{array}\right|^{t} \\
\overrightarrow{1} & \equiv\left|\begin{array}{llllll}
1 & 1 & \ldots & \ldots & 1 & \ldots
\end{array}\right|^{t}
\end{aligned}
$$

are two column vectors.

Thank to (11), (20), (29) we may write down a beautiful formula

$$
\left|\begin{array}{c}
1 \int_{0}^{n} S_{0}(x) \\
2 \int_{0}^{n} S_{1}(x) \\
3 \int_{0}^{n} S_{2}(x) \\
\ldots \\
m \int_{0}^{n} S_{m-1}(x)
\end{array}\right|=\left|\begin{array}{c}
S_{1}(n)-n B_{1} \\
S_{2}(n)-n B_{2} \\
S_{3}(n)-n B_{3} \\
\ldots \\
S_{m}(n)-n B_{m}
\end{array}\right|=P^{-1}\left|\begin{array}{c}
n^{2} \\
n^{3} \\
n^{4} \\
\ldots \\
n^{m+1}
\end{array}\right|
$$

\section{Generating Function of the Numbers $B_{m}$}

Although our method for obtaining $S_{m}(n)$ needs a set of numbers $B_{m}$ sufficiently well-defined from the formulae (19) and (24), we would like to clarify that they are indeed the well- known Bernoulli numbers.

To prove this assertion let us utilize (29) to get the matrix formula

$$
P \vec{B}+\overrightarrow{1}=0
$$

i.e. symbolically

$$
(P \vec{B}+\overrightarrow{1})_{m}=\sum_{k=1}^{m}\left(\begin{array}{c}
m+1 \\
k
\end{array}\right) B_{k}+1=:(B+1)^{m+1}-B^{m+1}=0
$$

where undefined notations $B^{k}$ must be replaced with $B_{k}$.

Knowing that

$$
e^{(B+1) t}-e^{B t}=\sum_{k=1}^{\infty}\left((B+1)^{k}-B^{k}\right) \frac{t^{k}}{k !}
$$

we see that the equation

$$
(B+1)^{k+1}-B^{k+1}=0 \quad k>0
$$

is equivalent to

$$
e^{(B+1) t}-e^{B t}=t
$$

On behalf of the above remark we may conclude that the numbers $B_{k}$ obey the relation

$$
\frac{t}{e^{t}-1}=e^{B t}=: \sum_{k=0}^{\infty} B_{k} \frac{t^{k}}{k !}
$$

as so as the symbolic relation (35).

As the Bernoulli numbers are defined by the same generating function (Beery 2010) from now on we may assimilate the numbers $B_{k}$ defined in (19), (24) with the Bernoulli number $B_{k}$.

5. Properties of $S_{m}(n)$ and $B_{k}$

(i) As resumes of the hereinabove results we cite the formulae (19), (21), (29)

$$
\begin{gathered}
B_{m+1}=-(m+1) \int_{0}^{1} S_{m}(x) \\
B_{m}=-\sum_{j=1}^{m} P_{m j}^{-1} \\
\vec{B}=-P^{-1} \overrightarrow{1}
\end{gathered}
$$

and (25) 


$$
B_{k}=\frac{2}{k+1} P_{k+11}^{-1}=\frac{2}{k+1} \frac{3}{k+2} P_{k+22}^{-1}=\ldots=\frac{(m+1) ! k !}{(k+m) !} P_{k+m m}^{-1}
$$

From (25) we have

$$
P_{m k}^{-1}=\frac{m !}{(k+1) !(m-k) !} B_{m-k}
$$

The formulae (14), (21), (38) lead to the well- known recurrence formulae

$$
B_{m}=-\sum_{k=1}^{m} \frac{m !}{(k+1) !(m-k) !} B_{m-k}=-\sum_{k=0}^{m-1} \frac{m !}{(m-k+1) ! k !} B_{k}
$$

i.e.

$$
\sum_{k=0}^{m} \frac{m !}{(m-k+1) ! k !} B_{k}=0
$$

as so as the formula linking $S_{m}(n)$ with the $B_{k}$

$$
\begin{aligned}
S_{m}(n) & =-\left(P_{m 1}^{-1}+P_{m 2}^{-1}+\ldots+P_{m m}^{-1}\right) n+P_{m 1}^{-1} n^{2}+\ldots+P_{m m}^{-1} n^{m+1} \\
S_{m}(n) & =B_{m} n+\frac{m}{2} B_{m-1} n^{2}+\ldots+\frac{1}{m+1} B_{0} n^{m+1} \\
& =\sum_{k=0}^{m} \frac{m !}{(k+1) !(m-k) !} B_{m-k} n^{k+1}
\end{aligned}
$$

(ii) An interesting property of $B_{k}$ cited in Coen (1996) has been proven by the remark that the function

$$
\frac{t}{e^{t}-1}+\frac{t}{2}=\frac{t}{2} \frac{e^{t}+1}{e^{t}-1}=\frac{t}{2} \operatorname{coth} \frac{t}{2}=\sum_{k=0}^{\infty} B_{k} \frac{t^{k}}{k !}+\frac{t}{2}
$$

is even so that

$$
B_{1}=-\frac{1}{2}
$$

and

$$
B_{2 k+1}=0 \quad \forall k \geq 1
$$

(iii) New relations between the numbers $B_{k}$ may be obtained from the property $P P^{-1} \equiv \hat{I}$.

In fact we see that the first column vector of $P^{-1}$

$$
\vec{C}_{1} \equiv\left|P^{-1}{ }_{11} P^{-1}{ }_{21} P^{-1}{ }_{31} \ldots . P^{-1}{ }_{n 1} \ldots .00\right|^{t}
$$

is perpendicular to the $j^{\text {th }}$ line vector $\vec{L}_{j}$ of $P$ for $1<j$ so that $\vec{C}_{1}$ is perpendicular with all combinations of the vectors $\vec{L}_{j}$ with $1<j$.

The first $\vec{L}_{j}$ are

$$
\begin{aligned}
& \vec{L}_{2} \equiv|33 \ldots \ldots \ldots . .| \\
& \vec{L}_{3} \equiv|464 \ldots \ldots \ldots . .| \\
& \cdots \\
& \vec{L}_{15} \equiv|1612056018204368800811440128701144080084368182056012016|
\end{aligned}
$$

The first interesting linear combinations of them and their implications are 


$$
\begin{aligned}
& |11 \ldots \ldots \ldots . . .| \Rightarrow P^{-1}{ }_{21}+P^{-1}{ }_{11}=0 \\
& |012 \ldots \ldots \ldots . . . .| \Rightarrow 2 P^{-1}{ }_{31}=-P^{-1}{ }_{21}=P^{-1}{ }_{11} \\
& |0001 \ldots \ldots \ldots . . . .| \Rightarrow P^{-1}{ }_{41}=0 \\
& |00103 \ldots \ldots \ldots . . . .| \Rightarrow 3 P^{-1}{ }_{51}=-P^{-1}{ }_{31} \\
& |000001 \ldots \ldots . . . .| \Rightarrow P_{61}^{-1}=0 \\
& |0000101 \ldots \ldots \ldots . .| \Rightarrow P_{71}^{-1}=-P_{51}^{-1} \\
& |00000001 \ldots \ldots \ldots . . .| \Rightarrow P_{81}^{-1}=0 \\
& |000000905 \ldots \ldots . .| \Rightarrow 5 P_{91}^{-1}=-9 P_{71}^{-1} \\
& |0000000001 \ldots \ldots . . .| \Rightarrow P_{101}^{-1}=0 \\
& |00000002509 \ldots \ldots . . .| \Rightarrow P_{111}^{-1}=-\frac{25}{9} P_{91}^{-1}=5 P_{71}^{-1}=\frac{5}{12} \\
& |000000000001 \ldots \ldots . .| \Rightarrow P_{121}^{-1}=0 \\
& |0000000000(691) 0(175) \ldots \ldots . . .| \Rightarrow P_{131}^{-1}=-\frac{691}{175} P_{111}^{-1}=-\frac{691}{420} \\
& |00000000000001 \ldots \ldots . .| \Rightarrow P_{141}^{-1}=0 \\
& |0000000000(-21) 0001 \ldots \ldots . .| \Rightarrow \quad P^{-1}{ }_{151}=21 P^{-1}{ }_{11}=\frac{35}{4} \\
& |0000000000000001 \ldots . . . .| \Rightarrow P_{161}^{-1}=0
\end{aligned}
$$

We may conclude that there exist relations between any two Bernoulli numbers via the relation $B_{m}=\frac{2}{m+1} P^{-1}{ }_{m+11}$ that we may calculate as shown in (46), for example

$$
B_{14}=\frac{2}{15} P_{151}^{-1}=\frac{2}{15} 21 P^{-1} 11=\frac{2}{15} 21 \frac{11}{2} B_{10}=\frac{77}{5} \frac{5}{66}=\frac{7}{6}
$$

\section{Remarks and Conclusion}

We think that this work is a noticeable contribution to the comprehension and the practical calculations of sums of powers of integers and the Bernoulli numbers via the inverse of the Pascal matrix, denoted herein by $P^{-1}$. The algorithm for calculating the matrix $P^{-1}$ needs few arithmetic operations. In fact, because nearly haft of the $B_{k}$ and the $P_{k m}^{-1}$ with $m<k$ are null and $P_{k k}^{-1}, P_{k k-1}^{-1}$ are known, one needs less than $(m-1)^{2} / 2$ additions/multiplications of rational numbers in order to obtain the $m$ first values of $B_{k}$ and $m$ fulfilled parallels with respect to the principal diagonal of $P^{-1}$. For example it needs only 35 such operations to get $B_{10}, S_{10}(n)$; 100 for 17 Faulhaber formulae; about 4.000 for $B_{100}, S_{100}(n)$.

For arriving to these results we are indebted to the antecedent works of many, many authors, especially of our great Pascal and of Rough M. who had the famous idea of integrating the equation $S_{m}(x+1)-S_{m}(x)=x^{m}$ from which we discover the relations between the universal matrix $P^{-1}$, the sums $S_{m}(n)$ and the set $B_{m}$.

The author is conscientious that there are properties of Bernoulli numbers it is interesting to be explored by the method discussed in this work, especially the theorem of Von Staudt-Clausen, the Kummer theorem, the Euler-Mclaurin formula, the relations between Bernoulli numbers and the zeta function, etc...one can find easily in literature.

The author dedicates this work to the memory of Nguyen Thai Binh, an example for a whole generation of abroad students like him fifty years ago.

\section{References}

Beery, J. (2010). Sums of Powers of Positive Integers - Introduction. Mathematical Association of America. Retrieved from http://www.maa.org/press/periodicals/convergence/sums-of-powers-of-positive-integers-introduction

Coen, L. E. S. (1996). Sums of powers and the Bernoulli numbers (Master's thesis, Eastern Illinois University). Retrieved from http://thekeep.eiu.edu/theses/1896

Edwards, A. W. F. (1986). A quick route to sums of powers. The American Mathematical Monthly, 93(6), 451-455.

Johnson, J. A. (1986). Summing the powers of the integers using calculus. The Mathematics Teacher, 79(3), 218-219. 
Knuth, D. E. (1993). Johann Faulhaber and sums of powers. Mathematics of Computation, 61(203), 277-294.

Raugh, M. (2014). The Bernoulli Summation Formula: A Pretty Natural Derivation. Ph.D. in Math, Stanford University for the 29th LACC High School Math Contest, March 22. Retrieved from www.mikeraugh.org

Si, D. T. (2016). Differential Calculus for Differential Equations, Special Functions, Laplace Transform, CCSE, Applied Physics Research, 8(1), 158-187. http://dx.doi.org/10.5539/apr.v8n1p158

Si, D. T. (2016). Operator calculus. Edification and Utilization. Lambert Academic Publishing.

\section{Appendix}

1) The 17 first Bernoulli numbers

$$
\left\{B_{0}, \ldots . ., B_{17}\right\} \equiv\left\{1,-\frac{1}{2}, \frac{1}{6}, 0,-\frac{1}{30}, 0, \frac{1}{42}, 0,-\frac{1}{30}, 0, \frac{5}{66}, 0, \frac{-691}{2730}, 0, \frac{7}{6}, 0,-\frac{3617}{510}, 0\right\}
$$

2) The $17 \times 17$ sub-matrix of the matrix $P^{-1}$

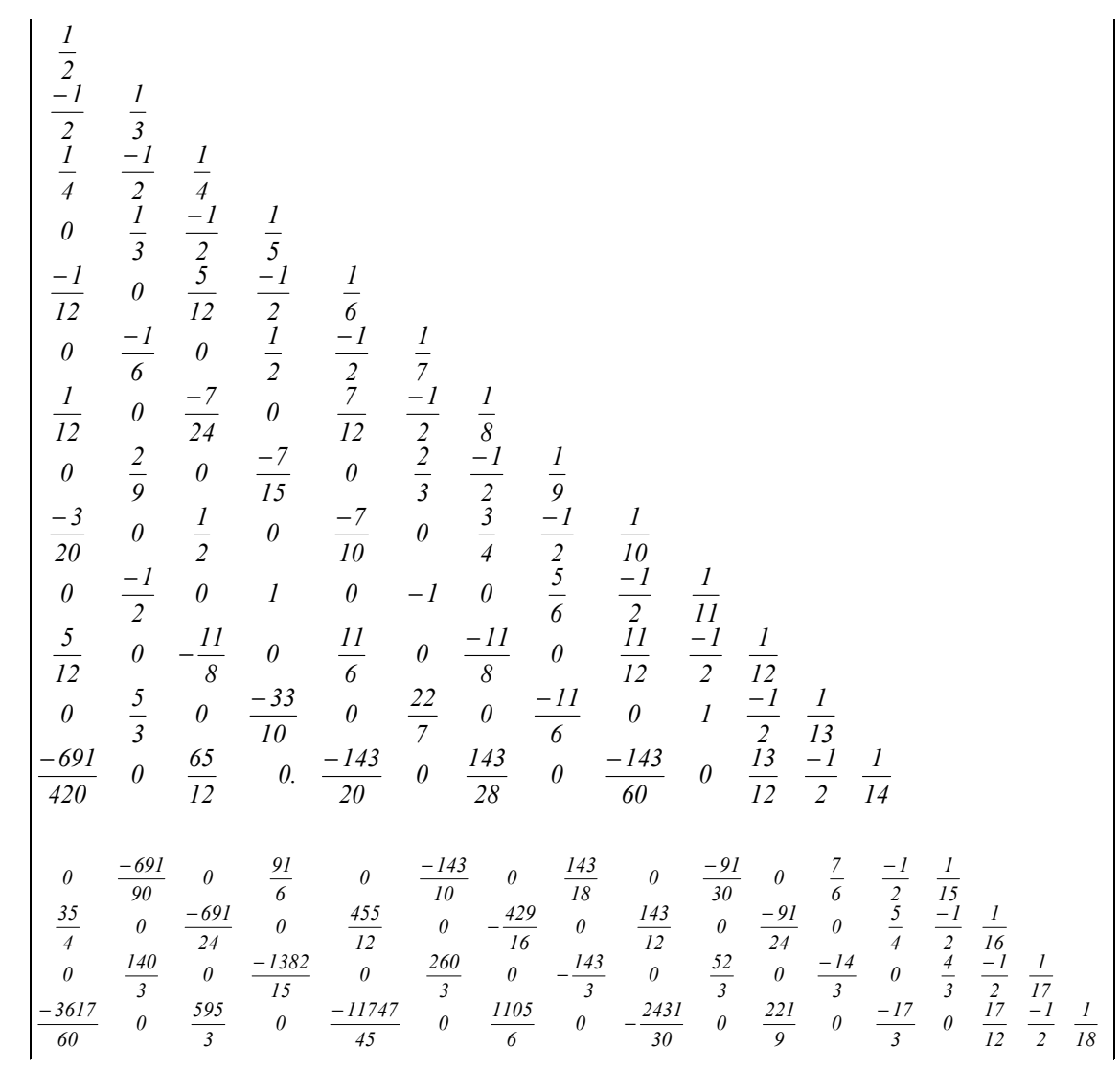

\section{Copyrights}

Copyright for this article is retained by the author(s), with first publication rights granted to the journal.

This is an open-access article distributed under the terms and conditions of the Creative Commons Attribution license (http://creativecommons.org/licenses/by/4.0/). 